\title{
The $a b$ initio model potential method: Third-series transition metal elements
}

\author{
Marcos Casarrubios and Luis Seijo ${ }^{\mathrm{a})}$ \\ Departamento de Química, C-XIV, Universidad Autónoma de Madrid, 28049 Madrid, Spain
}

(Received 20 July 1998; accepted 22 September 1998)

\begin{abstract}
In this paper we present nonrelativistic and relativistic core $a b$ initio model potentials (AIMPs) and valence basis sets for La and the third-series transition metal elements. The relativistic AIMPs are derived from atomic Cowan-Griffin calculations; they are made of a spin-free part and a one-electron spin-orbit operator according to Wood and Boring. The core potentials correspond to the 62-electron core $[\mathrm{Cd}, 4 f]$. The valence basis sets are optimized and spin-orbit corrected. We present monitoring spin-free calculations on the atoms, singly ionized ions and monohydrides of the ten elements, which show a good performance overall. A spin-free-state-shifted spin-orbit-configuration interaction calculation on $\mathrm{Pt}$, which uses empirical spin-free data and which is expected to be essentially free from spin-free deficiencies, points out that the quality of the spin-orbit operators is very good. (C) 1999 American Institute of Physics.
\end{abstract}

[S0021-9606(99)30301-9]

\section{INTRODUCTION}

Effective core potential (ECP) methods are widely accepted as efficient tools for reducing the computational demands of molecular and solid state $a b$ initio calculations without damaging the quality of the calculated valence properties. ${ }^{1,2}$ ECP methods are especially indicated for the heavy elements of the Periodic Table, since they have a large number of core electrons which can be safely frozen and, in addition, they demand the consideration of relativistic effects, which can be handled with economy and precision by means of ECP methods. ${ }^{3}$ In particular, several sets of ECPs exist for the third-series transition metal elements. Some of them are ultimately based upon the Phillips-Kleinman equation ${ }^{4}$ and rely on the pseudo-orbital transformation that produces nodeless valence pseudo-orbitals; this is the case of the pseudopotentials produced by Bachelet et al. ${ }^{5}$ Hay and Wadt, ${ }^{6,7}$ Ross et al. ${ }^{8}$ and Andrae et al. ${ }^{9}$ Some others are based on the Huzinaga-Cantu equation ${ }^{10}$ and lead to valence orbitals with the same nodal structure as the all-electron orbitals; this is the case of the model potentials produced by Sakai et al. ${ }^{11}$

Also based on the Huzinaga-Cantu equation, the $a b$ initio model potential (AIMP) ${ }^{12}$ method resulted from the implementation of two ideas which contrast with the basics of all the other ECP methods: (i) the core model potentials are obtained directly from the frozen core orbitals, without resorting to parametrization procedures based on the valence orbitals, and (ii) the components of the core model potentials must mimic the operators that they substitute as much as possible, while reducing the computing time. Accordingly, nonrelativistic AIMPs (NR-AIMP) and spin-free relativistic AIMPs derived from atomic Cowan-Griffin ${ }^{13}$ calculations (CG-AIMP) have been produced and successfully moni-

\footnotetext{
a) Author to whom correspondence should be addressed. Electronic mail: luis.seijo@uam.es
}

tored for the main-group elements, ${ }^{12,14,15}$ and for the firstseries and second-series transition metal elements, ${ }^{14,16,17}$ but they are not available for the third-series transition metal elements. Along a parallel line, spin-free relativistic AIMPs aimed to be used with the no-pair Douglas-Kroll ${ }^{18}$ Hamiltonian (NP-AIMP) have been produced for the third-series transition metal elements by Wittborn and Wahlgren, ${ }^{19}$ as well as for the transition metal elements from $\mathrm{Sc}$ to $\mathrm{Hg}$, altogether with optimized valence basis sets, by Rakowitz et $a l^{20}$

The ability of the spin-free CG-AIMPs to represent genuine relativistic effects in a consistent manner down to a group of the Periodic Table was shown in Ref. 15. An extension of the CG-AIMP method to include spin-orbit effects according to Wood-Boring's ideas ${ }^{21}$ was proposed and implemented (WB-AIMP), ${ }^{22,23}$ and the corresponding spinorbit operators and spin-orbit-corrected valence basis sets were produced for the main-group elements as well as for the first- and second-series transition metal elements. ${ }^{23,24}$ Also, a simple and efficient spin-free-state-shifting technique (sfss) was proposed to be used in spin-orbit-configuration interaction (CI) calculations in a basis of double-group symmetry adapted functions, as a practical means to decouple electron correlation and spin-orbit interactions, while including a significant amount of spin-orbit polarization. ${ }^{25}$ The use of the sfss technique with empirical spin-free spectra allows us to perform spin-orbit-CI calculations, essentially free of deficiencies in the treatment of correlation, which are ideal for monitoring the quality of spin-orbit operators; sfss-spinorbit-CI calculations on $\mathrm{Ir}^{+}$pointed out the very good quality of the WB-AIMP spin-orbit operators. ${ }^{26}$

In this work, we produced the ingredients of nonrelativistic NR-AIMP and of relativistic spin-free CG-AIMP and spin-orbit WB-AIMP calculations for La and the third-series transition metal elements: core model potentials, spin-orbitcorrected valence basis sets, and spin-orbit operators. We 
monitored their performance in spin-free calculations on the atoms, singly ionized ions, and monohydrides of the ten elements and in a spin-free-state-shifted spin-orbit CI calculation on the even spectrum of Pt. The overall quality of the results is good.

\section{METHOD: MODEL POTENTIALS AND VALENCE BASIS SETS}

The detailed procedures to follow in order to obtain the nonrelativistic AIMPs (NR-AIMP), the spin-free relativistic AIMPs derived from Cowan-Griffin atomic calculations (CG-AIMP), and the Wood-Boring one-electron spin-orbit operators which are added to the CG-AIMP Hamiltonian in order to produce the spin-dependent WB-AIMP Hamiltonian, as well as the corresponding valence basis sets, are fully described in Refs. 12, 17, and 23. Here we will only outline very briefly the procedure of the relativistic version.

\section{A. WB-AIMP Hamiltonian}

The spin-dependent relativistic WB-AIMP Hamiltonian of a molecule $H^{\mathrm{WB}-\mathrm{AIMP}}$ is the sum of a spin-free relativistic Hamiltonian $H^{\text {CG-AIMP }}$ and a pure spin-orbit Hamiltonian $H^{\mathrm{SO}}$ :

$$
H^{\mathrm{WB}-\mathrm{AIMP}}=H^{\mathrm{CG}-\mathrm{AIMP}}+H^{\mathrm{SO}} .
$$

For a molecule with $N_{\text {val }}$ valence electrons and $N_{\text {nuc }}$ nuclei (each with nuclear charge $Z^{I}$ and number of core electrons $Z_{\text {core }}^{I}$ ), the spin-free Hamiltonian reads

$$
\begin{aligned}
H^{\mathrm{CG}-\mathrm{AIMP}}= & \sum_{i}^{N_{\text {val }}} h^{\mathrm{CG}-\operatorname{AIMP}}(i)+\sum_{i<j}^{N_{\text {val }}} \frac{1}{r_{i j}} \\
& +\sum_{I<J}^{N_{\text {nuc }}} \frac{\left(Z^{I}-Z_{\text {core }}^{I}\right)\left(Z^{J}-Z_{\text {core }}^{J}\right)}{R_{I J}},
\end{aligned}
$$

with $h^{\text {CG-AIMP }}(i)$, the one-electron spin-free relativistic Cowan-Griffin $a b$ initio model potential operator, defined by

$$
\begin{aligned}
h^{\text {CG-AIMP }}(i)= & -\frac{1}{2} \nabla_{i}^{2}+\sum_{I}^{\text {nuclei }}\left[-\frac{Z^{I}-Z_{\text {core }}^{I}}{r_{i}}\right. \\
& +\sum_{k} \frac{C_{k}^{I} \exp \left(-\alpha_{k}^{I} r_{i}^{2}\right)}{r_{i}} \\
& +\sum_{l} \sum_{m=-l}^{+l} \sum_{a, b}\left|\chi_{a l m}^{I}\right\rangle A_{l ; a b}^{I, \mathrm{MP}}\left\langle\chi_{b l m}^{I}\right| \\
& \left.+\sum_{c}^{\text {core }}\left(-2 \varepsilon_{c}^{I}\right)\left|\phi_{c}^{I}\right\rangle\left\langle\phi_{c}^{I}\right|\right] .
\end{aligned}
$$

The terms in Eq. (3) are the nonrelativistic kinetic energy operator and a sum over the atoms in the molecule, which includes the operators of the nuclear attraction, core Coulomb and exchange interactions, and mass velocity plus Darwin interactions, as well as a term resulting from the linearindependency conditions between core and valence orbitals. For each atom $I$, the first term is the nuclear attraction fully shielded by the core electrons and the rest are the AIMP terms. They are obtained as follows: A numerical all-electron spin-free relativistic Cowan-Griffin-Hartree-Fock calculation ${ }^{13}$ is performed on a given state of atom $I$ (usually the ground state, see Table II). From this calculation, several atomic orbitals are arbitrarily chosen to be the core orbitals; they are represented by analytical Gaussian expansions which are obtained by a maximum overlap criterium $^{27}$ $\left(\phi_{1 s}-\phi_{5 s}, \phi_{2 p}-\phi_{4 p}, \phi_{3 d^{-}} \phi_{4 d}\right.$, and $\phi_{4 f}$ for the third-series transition metal elements in this paper, which we will call in short $[\mathrm{Cd}, 4 f])$, and they and their orbital energies $\left(\varepsilon_{1 s}-\varepsilon_{5 s}, \varepsilon_{2 p}-\varepsilon_{4 p}, \varepsilon_{3 d}-\varepsilon_{4 d}, \varepsilon_{4 f}\right)$ are conveniently stored in libraries. With the core orbitals, the core Coulomb potential $2 \sum \hat{J}_{c}(r)$ is calculated, the nuclear attraction corresponding to the core electrons $-Z_{\text {core }}^{I} / r$ is added to it $(-62 / r$ here), and the result is represented with a local potential $1 / r \sum_{k} C_{k} \exp \left(-\alpha_{k} r^{2}\right)$ by means of a least-squares fitting and it is stored. This is the third term on the right hand side of Eq. (3). The mass-velocity and Darwin radial numerical operators of Cowan-Griffin (not to be confused with Pauli's mass velocity and Darwin operators $)^{13}$ which correspond to the valence orbitals are also stored $\left[V_{\mathrm{MD}, 5 p}(r), V_{\mathrm{MD}, 5 d}(r)\right.$ and $V_{\mathrm{MD}, 6 s}(r)$, here]. In a given atomic or molecular calculation, these scalar relativistic operators are added to the core exchange operator $-\sum \hat{K}_{c}$ and the result is spectrally represented in the space defined by the set of (Gaussian) primitive functions used for atom $I,\left\{\chi_{a l m}^{I}\right\}$, which results in the fourth term on the right hand side of Eq. (3). In consequence, the $A_{l ; a b}^{I, \mathrm{MP}}$ coefficients are the elements of the product matrix $S^{I-1} V_{\mathrm{EMD}}^{I} S^{I-1}$; here, $S^{I}$ is the overlap matrix in the basis of primitives $\left\{\chi_{\text {alm }}^{I}\right\}$ and $V_{\mathrm{EMD}}^{I}$ is the matrix of the operator $-\Sigma \hat{K}_{c}+\hat{O}_{p} V_{M D, 5 p} \hat{O}_{p}+\hat{O}_{d} V_{M D, 5 d} \hat{O}_{d}+\hat{O}_{s} V_{M D, 6 s} \hat{O}_{s}$ of atom $I$ in the same basis $\left(\hat{O}_{l}=\Sigma_{m=-l}^{+l}|l m\rangle\langle l m|\right)$. Since the set of primitives $\left\{\chi_{a l m}^{I}\right\}$ is likely to change from one molecular calculation to another and it would not be efficient to tabulate the $A_{l ; a b}^{I, \mathrm{MP}}$ coefficients, they are instead calculated during the input processing part of every single molecular calculation. ${ }^{28}$ The last term on the right hand side of Eq. (3), which results from the linear-independency conditions between core and valence orbitals ${ }^{10}$ is calculated with the core orbitals and orbital energies. All this defines the spin-free CG-AIMP Hamiltonian, which is obtained without resorting to any parametrization procedure based on the use of the valence orbitals.

The ingredients of the spin-free relativistic CG-AIMPs corresponding to the frozen-core $[\mathrm{Cd}, 4 f]$ of the third-series transition metal elements have been produced in this work ([Cd] frozen-core for La): The sets of $\left\{C_{k}, \alpha_{k}\right\}$ parameters, the core orbitals $\phi_{1 s}-\phi_{5 s}, \phi_{2 p}-\phi_{4 p}, \phi_{3 d^{-}} \phi_{4 d}, \phi_{4 f}$, their orbital energies $\varepsilon_{1 s}-\varepsilon_{5 s}, \varepsilon_{2 p}-\varepsilon_{4 p}, \varepsilon_{3 d}-\varepsilon_{4 d}, \varepsilon_{4 f}$, and the radial mass-velocity plus Darwin operators $V_{M D, 5 p}(r), V_{M D, 5 d}(r)$ and $V_{M D, 6 s}(r)$. The corresponding nonrelativistic NR-AIMPs, which are necessary in order to study the size of the relativistic effects, have been produced as well (in this case the mass velocity and Darwin operators are suppressed). All these data are available from the authors. ${ }^{29}$ Valence basis sets, Coulomb model potentials and core orbitals are presented in a PAPS document. ${ }^{30}$

The spin-orbit contribution in Eq. (1) is 


$$
H^{\mathrm{SO}}=\sum_{i}^{N_{\text {val }}} \sum_{I}^{\text {nuclei }} h_{\mathrm{SO}}^{I}(i)
$$

with the atomic one-electron spin-orbit terms,

$$
h_{\mathrm{SO}}^{I}(i)=\lambda \sum_{n l}^{I} V_{\mathrm{SO}, n l}^{I, \mathrm{MP}}\left(r_{i}\right) \hat{O}_{l}^{I} \hat{l}^{\wedge} \hat{\boldsymbol{s}} \hat{O}_{l}^{I} \text {. }
$$

Herein, $\hat{\boldsymbol{l}}^{I}$ and $\hat{\boldsymbol{s}}$ are the usual vector angular momentum and spin operators, respectively; the projectors $\hat{O}_{l}^{I}$ (defined in terms of the spherical harmonics as $\hat{O}_{l}^{I}$ $\left.=\Sigma_{m=-l}^{+l}|l m, I\rangle\langle l m, I|\right)$ are used in the form proposed by Pitzer and Winter. ${ }^{31}$ The radial components are chosen to be analytical functions,

$$
V_{\mathrm{SO}, n l}^{I, \mathrm{MP}}\left(r_{i}\right)=\sum_{k} \frac{B_{k}^{n l, I} \exp \left(-\beta_{k}^{n l, I} r_{i}^{2}\right)}{r_{i}^{2}},
$$

whose parameters $\left\{B_{k}^{n l, I}, \beta_{k}^{n l, I}\right\}$ are determined through weighted least-squares fitting to the radial part of the WoodBoring spin-orbit operator ${ }^{21}$ which reads

$$
V_{\mathrm{SO}, n l}(r)=\frac{\alpha^{2}}{\left\{2+\alpha^{2}\left[\epsilon_{n l}-V(r)\right]\right\} r} \frac{d V(r)}{d r} .
$$

Herein, $\alpha$ is the fine-structure constant, $\epsilon_{n l}$ are the orbital energies of the spin-free relativistic equations of Cowan and Griffin, and $V(r)$ is an $X \alpha$ approximation to the HartreeFock (HF) one-electron potential. ${ }^{13}$ This effective oneelectron spin-orbit operator includes an average of twoelectron contributions through the use of the $X \alpha$ HartreeFock potential $V(r)$, although its detailed relationship to a mean-field spin-orbit operator is unknown. Also, an atomic scaling factor $\lambda^{I}$ is included in Eq. (5) which was first empirically parametrized ${ }^{23}$ and later found to be unnecessary; ${ }^{26}$ in consequence, we use $\lambda^{I}=1$. The $\left\{B_{k}, \beta_{k}\right\}$ parameters for the third-series transition metal elements have been produced here and they are presented in Table I.

\section{B. Spin-free-state-shifted WB-AIMP Hamiltonian}

The spin-free relativistic $H^{\text {CG-AIMP }}$ Hamiltonian [Eq. (2)] is used in standard nonrelativistic methods. The spindependent $H^{\text {WB-AIMP }}$ Hamiltonian [Eq. (1)] is used in spinorbit CI calculations, e.g., in a basis of double-group symmetry-adapted functions with HF or complete active space self-consistent field (CASSCF) orbitals produced with the spin-free $H^{\text {CG-AIMP }}$ Hamiltonian. In order to treat electron correlation and spin-orbit interactions at the highest possible level, the sfss Hamiltonian $H_{\text {sfss }}^{\mathrm{WB}-A I M P}$ was introduced: ${ }^{25,26}$

$$
\begin{aligned}
H_{\mathrm{sfss}}^{\mathrm{WB}-\mathrm{AIMP}}= & H^{\mathrm{WB}-\mathrm{AIMP}}+\sum_{i S M_{S} \Gamma \gamma} \delta(i S \Gamma)\left|\Phi^{\mathcal{P}}\left(i S M_{S} \Gamma \gamma\right)\right\rangle \\
& \times\left\langle\Phi^{\mathcal{P}}\left(i S M_{S} \Gamma \gamma\right)\right|
\end{aligned}
$$

with

$$
\delta(i S \Gamma)=\left[E^{\mathcal{G}}(i S \Gamma)-E^{\mathcal{G}}(\mathrm{GS})\right]-\left[E^{\mathcal{P}}(i S \Gamma)-E^{\mathcal{P}}(\mathrm{GS})\right] .
$$

Here, $\mathcal{P}$ and $\mathcal{G}$ are two CI spaces of, respectively, small and large relative size; $\mathcal{P}$ is supposed to be good enough for the calculation of the spin-orbit couplings but not for the electron correlation effects, for which the much larger $\mathcal{G}$ space is necessary. $\Phi^{\mathcal{P}}\left(i S M_{S} \Gamma \gamma\right)$ are spin-free CI wave functions in the small space. GS refers to the ground state, but it can be any given spin-free state. This sfss Hamiltonian is a practical means to take advantage of the fact that electron correlation is handled with a much larger efficiency with spin-free Hamiltonians than with spin-dependent Hamiltonians. Its use is based on the assumption that correlation and spin-orbit can be decoupled to a large extent. A sfss spin-orbit calculation requires performing correlated spin-free calculations with the $H^{\text {CG-AIMP }}$ Hamiltonian using the $\mathcal{G}$ and $\mathcal{P}$ spaces and one final spin-orbit CI calculation with the $H_{\text {sfss }}^{\text {WB-AIMP }}$ Hamiltonian using the $\mathcal{P}$ space. A $\mathcal{P}$ space made of the significant reference configurations plus single excitations which can partially take care of spin-orbit polarizations has been proven to be very efficient in $\operatorname{Ir}^{+} .{ }^{26}$

\section{Atomic valence basis sets}

The $H^{\text {CG-AIMP }}$ Hamiltonian [Eq. (2)] is used in atomic valence-only Hartree-Fock calculations and the valence basis sets (exponents and coefficients) are optimized by minimization of the valence SCF energy using standard allelectron methods. ${ }^{32}$ The resulting valence atomic orbitals are spin-orbit corrected by changing the value of the innermost coefficient (and renormalizing) in such a way that the spinorbit valence orbital coupling constants $\left(\zeta_{5 p}\right.$ and $\zeta_{5 d}$ here) have the same value when they are evaluated using the numerical Cowan-Griffin-Hartree-Fock atomic orbitals and using the analytical spin-orbit-corrected valence orbitals. ${ }^{23}$ This procedure has been shown to significantly improve the spin-orbit dependent properties at the same time that the quality of the bonding related properties is maintained. ${ }^{23}$ Following this procedure, we obtained in this work the spinorbit-corrected relativistic valence basis sets for the thirdseries transition metal elements. We obtained as well the nonrelativistic ones. All of them are available from the authors. ${ }^{29}$ The basis sets obtained in this work are minimal valence basis sets made of $13 s 9 p 8 d$ primitive Gaussian functions ( $13 s 9 p 7 d$ for La) contracted as [1/1/1]. When they are used in atomic and molecular calculations their flexibility can be enhanced with the release of the outermost primitives and the addition of appropriate functions, such as polarization ( $p$ and $f$ ) and diffuse $(d)$ functions. Furthermore, artificial effects in molecules due to insufficient two-center orthogonality between the molecular orbitals and the $4 f$ core orbital of the transition metal elements can be eliminated by extending the valence basis set in molecular calculations with the fully contracted $4 f$ core orbital itself; ${ }^{12}$ a single split of this orbital provides polarization of the $5 d$ orbital and makes unnecessary the addition of polarization $f$ functions. As we will see in Sec. IV, a recommended valence basis set results in $s$ and $d$ double-split, $p$ single-split, the addition of one $p$-polarization primitive function (from Ref. 32), one diffuse $d$ primitive function (from extrapolation of the lowest exponents), and the $4 f$ core orbital (a five-primitive con- 
TABLE I. Radial components of the spin-orbit operators [Eq. (6)].

\begin{tabular}{|c|c|c|c|c|c|}
\hline \multicolumn{2}{|c|}{ Lanthanum } & \multicolumn{2}{|c|}{ Hafnium } & \multicolumn{2}{|c|}{ Tantalum } \\
\hline \multicolumn{2}{|c|}{$\mathrm{V}_{\mathrm{SO}}(5 \mathrm{p})$} & \multicolumn{2}{|c|}{$\mathrm{V}_{\mathrm{SO}}(5 \mathrm{p})$} & \multicolumn{2}{|c|}{$\mathrm{V}_{\mathrm{SO}}(5 \mathrm{p})$} \\
\hline$\beta_{k}$ & $B_{k}$ & $\beta_{k}$ & $B_{k}$ & $\beta_{k}$ & $B_{k}$ \\
\hline 1062434.0 & 0.316284351 & 828377.0 & 0.298838027 & 840608.0 & 0.295090740 \\
\hline 113943.8 & 0.259445609 & 92047.30 & 0.259885928 & 93875.00 & 0.260778075 \\
\hline 17362.70 & 0.143824809 & 14720.05 & 0.158482339 & 14929.95 & 0.161947106 \\
\hline 2937.296 & 0.076265260 & 2519.611 & 0.084861639 & 2519.611 & 0.086167150 \\
\hline 408.8461 & 0.034413499 & 386.8130 & 0.039569368 & 386.8130 & 0.040070293 \\
\hline 49.68297 & 0.013636063 & 54.44665 & 0.016299951 & 54.67635 & 0.016448812 \\
\hline 5.182618 & 0.003714795 & 6.581837 & 0.005417598 & 6.722247 & 0.005539299 \\
\hline 0.4925039 & 0.000558879 & 0.7071441 & 0.000729291 & 0.7077581 & 0.000748354 \\
\hline
\end{tabular}

\begin{tabular}{cccccc}
\multicolumn{2}{c}{$\mathrm{V}_{\mathrm{SO}}(5 \mathrm{~d})$} & \multicolumn{2}{c}{$\mathrm{V}_{\mathrm{SO}}(5 \mathrm{~d})$} & \multicolumn{2}{c}{$\mathrm{V}_{\mathrm{SO}}(5 \mathrm{~d})$} \\
$\beta_{k}$ & $B_{k}$ & $\beta_{k}$ & $B_{k}$ & $\beta_{k}$ & $B_{k}$ \\
\hline 648783.0 & 0.356649850 & 348937.0 & 0.366814713 & 349416.0 & 0.364827965 \\
62450.70 & 0.246617397 & 32404.40 & 0.241792838 & 32681.90 & 0.243007244 \\
8133.880 & 0.122464582 & 4061.380 & 0.114948612 & 4131.870 & 0.116489903 \\
1160.066 & 0.052313001 & 546.6730 & 0.048181516 & 561.3760 & 0.049178174 \\
171.0242 & 0.021397030 & 74.73160 & 0.018693068 & 77.42550 & 0.019185962 \\
24.93041 & 0.008620685 & 10.54420 & 0.006313701 & 11.01060 & 0.006553580 \\
3.023650 & 0.002413158 & 1.733970 & 0.001565644 & 1.828700 & 0.001630219 \\
.3210655 & 0.000321494 & 0.1491840 & 0.000104793 & 0.1770230 & 0.000127945
\end{tabular}

\begin{tabular}{|c|c|c|c|c|c|}
\hline \multicolumn{2}{|c|}{ Tungsten } & \multicolumn{2}{|c|}{ Rhenium } & \multicolumn{2}{|c|}{ Osmium } \\
\hline \multicolumn{2}{|c|}{$\mathrm{V}_{\mathrm{SO}}(5 \mathrm{p})$} & \multicolumn{2}{|c|}{$\mathrm{V}_{\mathrm{SO}}(5 \mathrm{p})$} & \multicolumn{2}{|c|}{$\mathrm{V}_{\mathrm{SO}}(5 \mathrm{p})$} \\
\hline$\beta_{k}$ & $B_{k}$ & $\beta_{k}$ & $B_{k}$ & $\beta_{k}$ & $B_{k}$ \\
\hline 840410.0 & 0.292284394 & 922982.0 & 0.281975229 & 1009680.0 & 0.271456538 \\
\hline 94870.30 & 0.259941525 & 104721.7 & 0.263327714 & 116787.0 & 0.262151089 \\
\hline 15148.14 & 0.165055520 & 16373.70 & 0.173618991 & 18740.30 & 0.179499356 \\
\hline 2519.611 & 0.087553721 & 2634.738 & 0.091390804 & 3041.200 & 0.098671823 \\
\hline 386.8130 & 0.040559331 & 399.0759 & 0.041813094 & 448.7360 & 0.045308897 \\
\hline 54.68635 & 0.016648257 & 56.33963 & 0.017068254 & 61.88880 & 0.018306087 \\
\hline 6.772687 & 0.005616782 & 7.062068 & 0.005810721 & 7.638500 & 0.006213038 \\
\hline 0.7036291 & 0.000758001 & 0.7314891 & 0.000807641 & 0.7819500 & 0.000890679 \\
\hline
\end{tabular}

\begin{tabular}{lccccc}
\multicolumn{2}{c}{$\mathrm{V}_{\mathrm{SO}}(5 \mathrm{~d})$} & \multicolumn{2}{c}{$\mathrm{V}_{\mathrm{SO}}(5 \mathrm{~d})$} & \multicolumn{2}{c}{$\mathrm{V}_{\mathrm{SO}}(5 \mathrm{~d})$} \\
\multicolumn{1}{c}{$\beta_{k}$} & $B_{k}$ & $\beta_{k}$ & $B_{k}$ & $\beta_{k}$ & $B_{k}$ \\
\hline 349596.0 & 0.362893222 & 349585.0 & 0.360991782 & 351666.0 & 0.358665412 \\
32920.90 & 0.244148559 & 33134.30 & 0.245228476 & 33589.80 & 0.246486326 \\
4196.500 & 0.117967330 & 4257.350 & 0.119397667 & 4356.250 & 0.121139567 \\
575.2550 & 0.050147739 & 588.6360 & 0.051101293 & 608.0600 & 0.052268725 \\
79.99100 & 0.019670568 & 82.47830 & 0.020151892 & 85.90800 & 0.020728011 \\
11.44480 & 0.006791469 & 11.85660 & 0.007029825 & 12.45210 & 0.007309680 \\
1.911340 & 0.001686669 & 1.982920 & 0.001737394 & 2.094200 & 0.001820379 \\
0.2042350 & 0.000150781 & 0.2307140 & 0.000172843 & 0.2622830 & 0.000201494
\end{tabular}

\begin{tabular}{|c|c|c|c|c|c|}
\hline \multicolumn{2}{|c|}{ Iridium } & \multicolumn{2}{|c|}{ Platinum } & \multicolumn{2}{|c|}{ Gold } \\
\hline \multicolumn{2}{|c|}{$\mathrm{V}_{\mathrm{SO}}(5 \mathrm{p})$} & \multicolumn{2}{|c|}{$\mathrm{V}_{\mathrm{SO}}(5 \mathrm{p})$} & \multicolumn{2}{|c|}{$\mathrm{V}_{\mathrm{SO}}(5 \mathrm{p})$} \\
\hline$\beta_{k}$ & $B_{k}$ & $\beta_{k}$ & $B_{k}$ & $\beta_{k}$ & $B_{k}$ \\
\hline 882208.0 & 0.280937582 & 938208.0 & 0.273778825 & 917162.0 & 0.273090953 \\
\hline 101270.3 & 0.260624153 & 107472.2 & 0.263432589 & 105793.0 & 0.262044288 \\
\hline 16368.63 & 0.172573920 & 17116.37 & 0.177544734 & 17107.55 & 0.177122614 \\
\hline 2730.553 & 0.093356420 & 2830.690 & 0.095918641 & 2876.224 & 0.096774473 \\
\hline 421.5071 & 0.043691874 & 437.5741 & 0.044861087 & 450.9637 & 0.045667731 \\
\hline 60.06378 & 0.017976984 & 62.81186 & 0.018533717 & 65.82322 & 0.019027479 \\
\hline 7.658918 & 0.006217620 & 8.084998 & 0.006485347 & 8.649507 & 0.006772759 \\
\hline 0.7905910 & 0.000912627 & 0.8363480 & 0.000986817 & 0.9189230 & 0.001100221 \\
\hline
\end{tabular}


TABLE I. (Continued.)

\begin{tabular}{|c|c|c|c|c|c|}
\hline \multicolumn{2}{|c|}{ Iridium } & \multicolumn{2}{|c|}{ Platinum } & \multicolumn{2}{|c|}{ Gold } \\
\hline \multicolumn{2}{|c|}{$\mathrm{V}_{\mathrm{SO}}(5 \mathrm{~d})$} & \multicolumn{2}{|c|}{$\mathrm{V}_{\mathrm{SO}}(5 \mathrm{~d})$} & \multicolumn{2}{|c|}{$\mathrm{V}_{\mathrm{SO}}(5 \mathrm{~d})$} \\
\hline$\beta_{k}$ & $B_{k}$ & $\beta_{k}$ & $B_{k}$ & $\beta_{k}$ & $B_{k}$ \\
\hline 351444.0 & 0.356798362 & 352227.0 & 0.354828824 & 522618.0 & 0.321613769 \\
\hline 33772.00 & 0.247464382 & 34052.30 & 0.248520440 & 55150.30 & 0.259216658 \\
\hline 4412.710 & 0.122498426 & 4483.450 & 0.123985135 & 8094.750 & 0.146062835 \\
\hline 621.0900 & 0.053200728 & 636.3300 & 0.054206909 & 1304.760 & 0.069573800 \\
\hline 88.39700 & 0.021209766 & 91.27500 & 0.021727345 & 221.7660 & 0.029945002 \\
\hline 12.86740 & 0.007551498 & 13.36150 & 0.007811745 & 41.54280 & 0.012869195 \\
\hline 2.161890 & 0.001871219 & 2.244970 & 0.001935926 & 6.959130 & 0.005412024 \\
\hline 0.2882640 & 0.000224480 & 0.3170000 & 0.000250328 & 0.8208680 & 0.000930658 \\
\hline \multicolumn{2}{|c|}{ Mercury } & & & & \\
\hline \multicolumn{2}{|c|}{$\mathrm{V}_{\mathrm{SO}}(5 \mathrm{p})$} & & & & \\
\hline$\beta_{k}$ & $B_{k}$ & & & & \\
\hline 932177.0 & 0.269394517 & & & & \\
\hline 108157.3 & 0.261683193 & & & & \\
\hline 17571.02 & 0.179717150 & & & & \\
\hline 2948.894 & 0.098863660 & & & & \\
\hline 461.5352 & 0.046780200 & & & & \\
\hline 67.10545 & 0.019465947 & & & & \\
\hline 8.823955 & 0.006938324 & & & & \\
\hline 0.9228180 & 0.001127373 & & & & \\
\hline \multicolumn{2}{|c|}{$\mathrm{V}_{\mathrm{SO}}(5 \mathrm{~d})$} & & & & \\
\hline$\beta_{k}$ & $B_{k}$ & & & & \\
\hline 353312.0 & 0.350677226 & & & & \\
\hline 34598.30 & 0.250421955 & & & & \\
\hline 4626.050 & 0.126897965 & & & & \\
\hline 667.3400 & 0.056246575 & & & & \\
\hline 97.07800 & 0.022780150 & & & & \\
\hline 14.35010 & 0.008338649 & & & & \\
\hline 2.401020 & 0.002066076 & & & & \\
\hline 0.3737860 & 0.000301015 & & & & \\
\hline
\end{tabular}

tracted function) single-split, that is a $13 s 10 p 9 d 5 f$ primitive set contracted as [3/3/4/2]. Further uncontraction leads to even more flexible basis sets. For Lanthanum, the $d$ splitting is a single one and the $f$ function has only one primitive from Ref. 33, that is a $13 s 10 p 8 d 1 f$ primitive set contracted as $[3 / 3 / 3 / 1]$.

\section{ATOMIC CALCULATIONS}

\section{A. Spin-free calculations}

In Table II we present spin-free relativistic CowanGriffin-Hartree-Fock valence energies and $5 p, 5 d$ and $6 s$ orbital energies and radial expectation values of the thirdseries transition metal elements. We show the results of the CG-AIMP calculations corresponding to the uncorrected and the spin-orbit corrected valence basis sets, together with the all-electron numerical Cowan-Griffin-Hartree-Fock calculations. ${ }^{13}$ We present as well the $5 p$ and $5 d$ spin-orbit coupling constants, defined as $\zeta_{n l}=\left\langle\phi_{n l}\left|V_{\mathrm{SO}, n l}\right| \phi_{n l}\right\rangle$; the allelectron numerical ones are calculated with the numerical spin-orbit operators [Eq. (7)] and the CG-AIMP ones with the analytical approximations of them [Eq. (6)]. It is observed that the spin-orbit correction of the basis sets, de- signed to produce correct orbital spin-orbit coupling constants, significantly improves the expectation value of $1 / r^{3}$, very much related to spin-orbit coupling, at a time that keeps essentially unaffected the orbital energies and the other radial expected values, which are bond-related properties. The overall agreement between the CG-AIMP results which use spin-orbit-corrected basis sets and the all-electron calculations is very good. We should note that, in contrast with pseudopotential methods, neither these properties nor the orbital shapes enter fitting procedures in the AIMP method; this agreement does simply reflect the facts that the model potentials efficiently mimic the operators substituted by them and that the valence basis sets are of good quality. A similar agreement is reached as well in the nonrelativistic case.

In Table III we present $6 s \rightarrow 5 d$ excitation energies and $6 s$ ionization energies, calculated at the Hartree-Fock level, which are useful to compare AIMP and all-electron results. The all-electron calculations are numerical. The basis sets used in the AIMP calculations result in triple- $s$, single- $p$ and double- $d$ split of the atomic orbitals and the addition of one polarization $p$ function ${ }^{32}$ and one diffuse $d$ function required for a proper description of the configurations with different $6 s$ and $5 d$ population $^{34}$ (obtained by extrapolation from the 
TABLE II. Spin-free relativistic valence energy, orbital energies and radial expectation values (in a.u.) and orbital spin-orbit coupling constants $\zeta_{n l}\left(\right.$ in $\mathrm{cm}^{-1}$ ). First entry: CG-AIMP calculations with the uncorrected valence basis sets. Second entry: CG-AIMP calculations with spin-orbit-corrected valence basis sets. Third entry: All-electron numerical Cowan-Griffin-Hartree-Fock calculations.

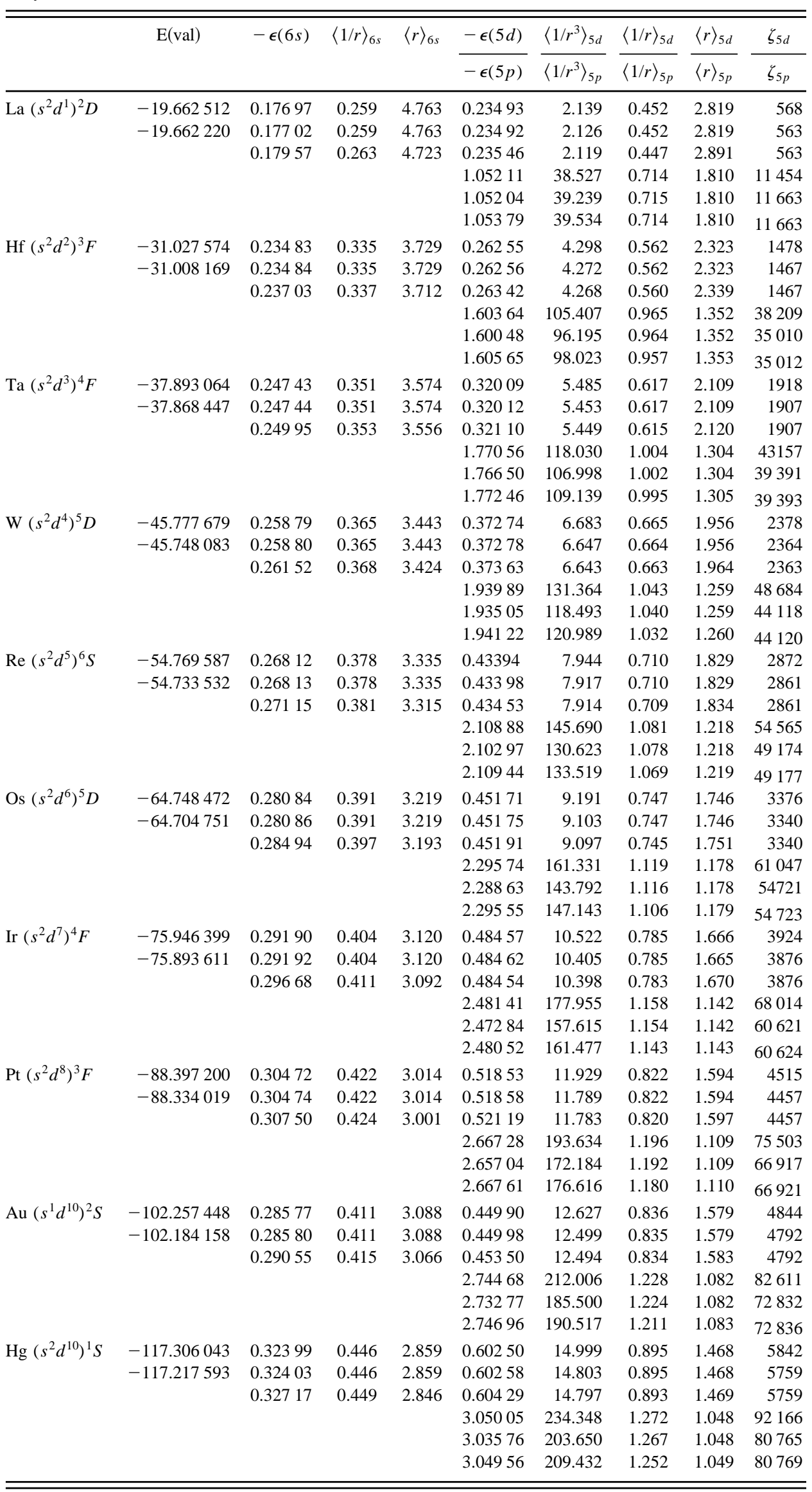


TABLE III. Atomic excitation and ionization energies with respect to the lowest atomic terms of the configurations $5 d^{n} 6 s^{2}$ : All-electron (first entry) and AIMP (second entry) nonrelativistic Hartree-Fock and spin-free relativistic Cowan-Griffin-Hartree-Fock results. No-pair spin-free relativistic results from Ref. 19 are included for comparison. All numbers in eV.

\begin{tabular}{lcrrrllll}
\hline \hline & NR-HF $^{\mathrm{a}}$ & CG-HF $^{\mathrm{b}}$ & NP-HF $^{\mathrm{c}}$ & Exp. $^{\mathrm{d}}$ & & NR-HF $^{\mathrm{a}}$ & CG-HF $^{\mathrm{b}}$ & Exp. $^{\mathrm{d}}$ \\
\hline$\rightarrow \mathrm{La}\left(s^{1} d^{2}\right)^{4} F$ & -0.98 & -0.30 & -0.31 & 0.36 & $\rightarrow \mathrm{La}^{+}\left(s^{1} d^{1}\right)^{3} D$ & $4.30^{\mathrm{e}}$ & $4.49^{\mathrm{e}}$ & 5.87 \\
& -0.94 & -0.30 & -0.38 & & & 4.32 & 4.50 & \\
$\rightarrow \mathrm{Hf}\left(s^{1} d^{3}\right)^{5} F$ & -0.38 & 0.95 & 0.94 & 1.69 & $\rightarrow \mathrm{Hf}^{+}\left(s^{1} d^{2}\right)^{4} F$ & 5.07 & 5.61 & - \\
& -0.36 & 0.95 & 0.91 & & & 5.10 & 5.62 & \\
$\rightarrow \mathrm{Ta}\left(s^{1} d^{4}\right)^{6} D$ & -1.28 & 0.21 & 0.18 & 1.04 & $\rightarrow \mathrm{Ta}^{+}\left(s^{1} d^{3}\right)^{5} F$ & 5.13 & 5.71 & 7.90 \\
& -1.27 & 0.19 & 0.16 & & & 5.16 & 5.72 & \\
$\rightarrow \mathrm{W}\left(s^{1} d^{5}\right)^{7} S$ & -2.95 & -1.29 & -1.31 & -0.18 & $\rightarrow \mathrm{W}^{+}\left(s^{1} d^{4}\right)^{6} D$ & 5.17 & 5.77 & 7.94 \\
& -2.93 & -1.26 & -1.35 & & & 5.21 & 5.81 & \\
$\rightarrow \operatorname{Re}\left(s^{1} d^{6}\right)^{6} D$ & -0.04 & 1.76 & 1.71 & 1.76 & $\rightarrow \operatorname{Re}^{+}\left(s^{1} d^{5}\right)^{7} S$ & 5.19 & 5.79 & 7.88 \\
& -0.03 & 1.73 & 1.69 & & & 5.24 & 5.80 & \\
$\rightarrow \mathrm{Os}\left(s^{1} d^{7}\right)^{5} F$ & -1.63 & 0.55 & 0.54 & 0.75 & $\rightarrow \mathrm{Os}^{+}\left(s^{1} d^{6}\right)^{6} D$ & 5.58 & 6.40 & 8.77 \\
& -1.61 & 0.52 & 0.50 & & & 5.62 & 6.41 & \\
$\rightarrow \operatorname{Ir}\left(s^{1} d^{8}\right)^{4} F$ & -2.43 & 0.09 & 0.07 & 0.40 & $\rightarrow \mathrm{Ir}^{+}\left(s^{1} d^{7}\right)^{5} F$ & 5.94 & 6.97 & - \\
& -2.41 & 0.07 & 0.00 & & & 5.96 & 6.97 & \\
$\rightarrow \operatorname{Pt}\left(s^{1} d^{9}\right)^{3} D$ & $-3.15^{\mathrm{e}}$ & -0.40 & -0.45 & -0.64 & $\rightarrow \mathrm{Pt}^{+}\left(s^{1} d^{8}\right)^{4} F$ & 6.26 & 7.51 & 9.22 \\
& -3.17 & -0.41 & -0.56 & & & 6.28 & 7.51 & \\
$\rightarrow \mathrm{Au}\left(s^{1} d^{10}\right)^{2} S$ & -5.13 & -1.86 & $\cdots$ & -1.74 & $\rightarrow \mathrm{Au}^{+}\left(s^{1} d^{9}\right)^{3} D$ & 6.56 & 8.03 & 9.76 \\
& -5.27 & -1.88 & $\cdots$ & & & 6.64 & 8.09 & \\
& $\cdots$ & $\cdots$ & $\cdots$ & $\ldots$ & $\rightarrow \mathrm{Hg}^{+}\left(s^{1} d^{10}\right)^{2} S$ & 6.83 & 8.51 & 10.43 \\
& $\cdots$ & $\cdots$ & $\cdots$ & $\ldots$ & & 6.84 & 8.47 & \\
\hline \hline
\end{tabular}

${ }^{a}$ Nonrelativistic Hartree-Fock calculations. AE results from Ref. 58.

${ }^{\mathrm{b}}$ Spin-free relativistic Cowan-Griffin-Hartree-Fock calculations (see Ref. 13). AE results from Ref. 58.

${ }^{\mathrm{c} N o-p a i r}$ spin-free relativistic Hartree-Fock calculations (Ref. 18) performed in Ref. 19.

${ }^{\mathrm{d}}$ Reference 58, averages over experimental spin-orbit components of Ref. 37.

'This work. This result is not coincident with that of Ref. 58.

two outermost exponents). The contraction used in the AIMP calculations is [4/3/4] ([4/3/3] for La upon single-d split only). Table III reveals that the deviations brought about by the AIMP approximations are small and that they are very similar in the nonrelativistic and in the spin-free relativistic calculations. The all-electron relativistic effects are, in consequence, very well reproduced in the AIMP calculations. The results of the spin-free relativistic Douglas-Kroll nopair calculations ${ }^{18}$ on the $6 s \rightarrow 5 d$ excitation energies of Ref. 19 have been included in Table III; one can observe that the ability of the AIMP approach to mimic the all-electron (AE) results is similar in this case, too. Also, the results of spinfree relativistic approximations of Douglas-Kroll and Cowan-Griffin are remarkably close, both at the all-electron and at the AIMP levels.

Once we know that the AIMP results resemble the AE ones within reasonable limits, and taking into account that electron correlation effects are necessary for a correct description of the atomic excitations under consideration, we performed CASSCF calculations ${ }^{35}$ where the active orbital space included the $5 d$ and $6 s$ orbitals, followed by average coupled-pair functional (ACPF) calculations ${ }^{36}$ in which the previous CASSCF space was used as a multireference for single and double excitations. In all these CASSCF and ACPF calculations, we double split rather than triple split the $s$ atomic orbital and we augmented the basis set with the $4 f$ core orbital singly split, which resulted in a final contraction [3/3/4/2], except for La where a [3/3/3] contraction was used. In a first set of ACPF calculations labeled ACPF-ds, we correlated only the $5 d$ and $6 s$ electrons; in a second set labeled
ACPF- $[\mathrm{p}] \mathrm{ds}$, we also allowed for single and double excitations from the $5 p$ closed shell. We performed all these calculations with the nonrelativistic and the spin-free relativistic Hamiltonians, NR-AIMP and CG-AIMP. The results are shown in Table IV. One can see that the correlation effects are significant in general. The relativistic effects are, however, crucial to reproduce the experimental values of these transitions. This is evident in Fig. 1, where the overall good quality of the CG-AIMP ACPF-[p]ds results is also clear.

\section{B. Spin-orbit calculations}

A realistic check of the quality of any spin-orbit operator can only be achieved through calculations that do not show any deficiencies in the treatment of spin-free effects. In particular, contaminations associated with insufficient treatment of electron correlation must be avoided. Recent calculations of $\mathrm{Ir}^{+}$(Ref. 26) have shown that electron correlation and spin-orbit effects can be effectively decoupled to a large extent by means of the spin-free-state-shifting technique [Eqs. (8) and (9)] and pointed out an unambiguous, systematic way to ascertain the accuracy of any spin-orbit operator based on the use of spin-free empirical information ${ }^{26}$ (or, alternatively, benchmark spin-free calculations). Here we monitored the quality of the spin-orbit operator of Pt accordingly, that is in a sfss-WB-AIMP multireference CI $[\mathrm{MRCI}(\mathrm{S})]$ spin-orbit calculation, as we describe next.

We used the $H_{\mathrm{sfss}}^{\mathrm{WB}-\mathrm{AIMP}}$ Hamiltonian [Eqs. (8) and (9)] with empirical data for the spin-free spectrum $E^{\mathcal{G}}(i S \Gamma)$ $-E^{\mathcal{G}}(\mathrm{GS})$ in a spin-orbit $\mathrm{MRCI}(\mathrm{S})$ calculation on the basis 
TABLE IV. Atomic excitation energies with respect to the lowest atomic terms of the configurations $5 d^{n} 6 s^{2}$. Nonrelativistic (first entry) and spinfree Cowan-Griffin relativistic (second entry) $a b$ initio model potential CASSCF and ACPF results. All numbers in eV.

\begin{tabular}{|c|c|c|c|c|c|}
\hline & & CASSCF $^{\mathrm{a}}$ & $A C P F-d s^{b}$ & $\mathrm{ACPF}-[\mathrm{p}] \mathrm{ds}{ }^{\mathrm{c}}$ & Exp. ${ }^{d}$ \\
\hline \multirow[t]{2}{*}{$\rightarrow \mathrm{La}\left(s^{1} d^{2}\right)^{4} F$} & NR-AIMP & -0.35 & - & 0.22 & \\
\hline & CG-AIMP & 0.13 & - & 0.73 & 0.36 \\
\hline \multirow[t]{2}{*}{$\rightarrow \mathrm{Hf}\left(s^{1} d^{3}\right)^{5} F$} & NR-AIMP & -0.24 & 0.34 & 0.36 & \\
\hline & CG-AIMP & 1.13 & 1.59 & 1.58 & 1.69 \\
\hline \multirow[t]{2}{*}{$\rightarrow \mathrm{Ta}\left(s^{1} d^{4}\right)^{6} D$} & NR-AIMP & -1.11 & -0.54 & -0.46 & \\
\hline & CG-AIMP & 0.38 & 0.82 & 0.86 & 1.04 \\
\hline \multirow[t]{2}{*}{$\rightarrow \mathrm{W}\left(s^{1} d^{5}\right)^{7} S$} & NR-AIMP & -2.70 & -1.99 & -1.85 & \\
\hline & CG-AIMP & -1.05 & -0.54 & -0.43 & -0.18 \\
\hline \multirow[t]{2}{*}{$\rightarrow \operatorname{Re}\left(s^{1} d^{6}\right)^{6} D$} & NR-AIMP & -0.03 & 0.01 & 0.22 & \\
\hline & CG-AIMP & 1.73 & 1.85 & 1.93 & 1.76 \\
\hline \multirow[t]{2}{*}{$\rightarrow \mathrm{Os}\left(s^{1} d^{7}\right)^{5} F$} & NR-AIMP & -1.61 & -1.40 & -1.25 & \\
\hline & CG-AIMP & 0.53 & 0.66 & 0.80 & 0.75 \\
\hline \multirow[t]{2}{*}{$\rightarrow \operatorname{Ir}\left(s^{1} d^{8}\right)^{4} F$} & NR-AIMP & -2.40 & -2.35 & -2.18 & \\
\hline & CG-AIMP & 0.07 & 0.06 & 0.23 & 0.40 \\
\hline \multirow[t]{2}{*}{$\rightarrow \operatorname{Pt}\left(s^{1} d^{9}\right)^{3} D$} & NR-AIMP & -3.17 & -3.25 & -3.04 & \\
\hline & CG-AIMP & -0.41 & -0.55 & -0.34 & -0.64 \\
\hline \multirow[t]{2}{*}{$\rightarrow \mathrm{Au}\left(s^{1} d^{10}\right)^{2} S$} & NR-AIMP & -5.27 & -5.45 & -5.18 & \\
\hline & CG-AIMP & -1.88 & -2.07 & -1.79 & -1.74 \\
\hline
\end{tabular}

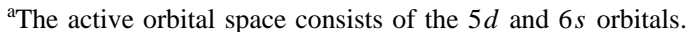

${ }^{\mathrm{b}} \mathrm{ACPF}$ calculation with a multireference which is the previous CASSCF. Only $5 d$ and $6 s$ electrons are correlated.

${ }^{\mathrm{c}} \mathrm{ACPF}$ calculation with a multireference which is the previous CASSCF. $5 p, 5 d$ and $6 s$ electrons are correlated.

${ }^{\mathrm{d}}$ Reference 58, averages over experimental spin-orbit components of Ref. 37.

of double-group symmetry-adapted functions which resulted after allowing single excitations from a multireference made of the relevant configurations: 21 configurations corresponding to the distribution of ten electrons in the $5 d$ and $6 s$ orbitals. (The orbitals were optimized in CG-AIMP HF calculations on $5 d^{8} 6 s^{2}-{ }^{3} \mathrm{~F}$. We used $\bar{D}_{2 h}$ double-group symmetry but obtained degeneracies with energy separations below $10^{-6}$ hartree for the components of a given $J$ quantum number.) In order to produce the empirical spin-free spectrum, we performed a generalized least-squares fitting of all the parameters in the interaction matrices to the experimental even spectrum of Pt (Ref. 37) using the programs of Cowan $^{38}$ and following the procedure described by Kleef and Metsch ${ }^{39}$ then, we diagonalized the interaction matrices which resulted from using all the spin-free parameters and setting all the spin-dependent ones to zero. The empirical spin-free spectrum that we obtained is shown in Table V. The results of the spin-free CG-AIMP $\mathrm{MRCI}(\mathrm{S})$ calculation performed in the same CI space used in the corresponding spin-orbit MRCI(S) and the corresponding shifting constants $\delta(i S \Gamma)[$ Eq. (9)] are also shown.

In Table VI we compare the results of the sfss-WBAIMP MRCI(S) spin-orbit calculation described above with the experiment. We can see that the quality of the results is very high, in line with what has already been observed in $\mathrm{Ir}^{+} .{ }^{26}$ This should be taken as an indication of two things: first, the quality of the spin-orbit operator is very high, and second, the spin-orbit and correlation effects can be decoupled to a large extent so that the latter can be handled in a separate spin-free calculation. We included in Table VI the

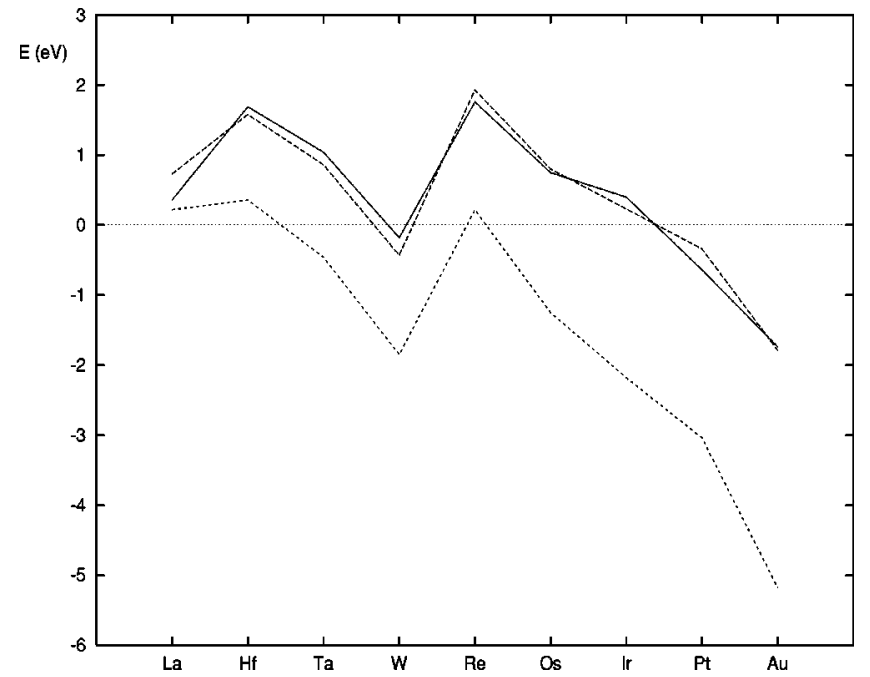

FIG. 1. $6 s \rightarrow 5 d$ transitions of the third-series transition metal elements: (full line) experimental data (averages over experimental spin-orbit components); (dashed line) spin-free relativistic CG-AIMP ACPF-[p]ds calculations of this work; (dotted line) nonrelativistic NR-AIMP ACPF-[p]ds calculations of this work.

results of an (unshifted) WB-AIMP MRCI(SD) calculation, in which a much larger CI space including also double excitations from the same reference was used, ${ }^{40}$ as well as the results of a Dirac-Hartree-Fock plus four-component CI (singles and doubles) calculation of Visscher et al. ${ }^{41}$ of a comparable level in the treatment of correlation. The results of both of them are quite close and show a reasonable agreement with the experiment, but significantly poorer than the sfss-WB-AIMP MRCI(S) calculation; what emerges from the comparison is that the main source of the deviations from the experiment in the two unshifted calculations is not the treatment of the spin-orbit effects but the treatment of the correlation effects, which indirectly contaminates the spinorbit splittings.

\section{MOLECULAR CALCULATIONS}

\section{A. Details of the calculations}

In this section we present the results of monitoring molecular calculations on $\mathrm{MH}$ monohydrides of the third-series transition metal elements. We performed CASSCF calculations in which the outermost M- $5 d$ and M- $6 s$ electrons and the $\mathrm{H}-1 s$ electron are distributed in all possible ways among the $\sigma, \sigma^{\prime}, \sigma^{\prime \prime}, \pi$ and $\delta$ molecular orbitals with main character of the above mentioned atomic orbitals. Using the CASSCF molecular orbitals we performed two sets of ACPF calculations (multireference single and double CI with sizeconsistency corrections $)^{36}$ with the CASSCF multireference: ACPF-ds, with single and double excitations from the active molecular orbitals, and ACPF-[p]ds, in which we correlated as well the $\mathrm{M}-5 p$ electrons by including all single and double excitations from the closed-shell of main character M-5 . Except when otherwise indicated, we have used for the metals the [3/3/4/2] valence basis set as explained below. For hydrogen, we used the $[6 \mathrm{~s}]$ set of Huzinaga ${ }^{42}$ augmented with two $p$ functions and contracted as [4/2]. We calculated dissociation energies with separate atomic calculations on 
TABLE V. Spin-free spectrum of Pt and parameters of the sfss spin-orbit Hamiltonian [Eqs. (8) and (9)]. All numbers in $\mathrm{cm}^{-1}$.

\begin{tabular}{lcrc}
\hline \hline Term & Empirical & $\begin{array}{c}\text { CG-AIMP } \\
\text { MRCI(S) }\end{array}$ & \multicolumn{1}{c}{$\delta(i S \Gamma)^{\mathrm{a}}$} \\
\hline${ }^{3} \mathrm{D}$ & 0 & 0 & 0 \\
$\mathrm{a}{ }^{1} \mathrm{~S}$ & 2435 & 797 & 1638 \\
$\mathrm{a}^{1} \mathrm{D}$ & 1632 & 2143 & -511 \\
${ }^{3} \mathrm{~F}$ & 3944 & 5591 & -1647 \\
${ }^{3} \mathrm{P}$ & 12457 & 17007 & -4550 \\
$\mathrm{~b}^{1} \mathrm{D}$ & 16646 & 20538 & -3892 \\
${ }^{1} \mathrm{G}$ & 17030 & 22118 & -5088 \\
$\mathrm{~b}^{1} \mathrm{~S}$ & 39846 & 48402 & -8556 \\
\hline \hline
\end{tabular}

${ }^{\mathrm{a} S e e ~ E q . ~(9) . ~}$

the ground states of $\mathrm{H}$ and $\mathrm{M}$, at the same level of wave function and basis set as the corresponding molecular calculation.

\section{B. Results}

First, in order to decide upon a recommendable pattern of the valence basis set, we compare the spectroscopic constants of the ${ }^{2} \Sigma^{+}$state of PtH calculated with CASSCF wave functions at the all-electron and AIMP levels in Table VII. These calculations were done nonrelativistically since variational all-electron Cowan-Griffin calculations cannot be performed in molecules, but the conclusions might be safely extended to the spin-free relativistic case. The basis sets used for Pt in the valence-only NR-AIMP calculations are different contraction schemes of the $13 s 10 p 9 d 5 f$ primitive set described in Sec. IIC and used in the CASSCF and ACPF calculations of Sec. III. The basis set used for Pt in the allelectron calculation was a $22 s 16 p 13 d 8 f$ primitive set of Faegri $^{43}$ augmented with the same $p$-polarization and $d$-diffuse functions as the valence-only calculation and contracted as [8/6/6/3]. The basis set of hydrogen described above was used in all the calculations. We observe in Table VII that the NR-AIMP calculation with the AE basis set produces results essentially coincident with the AE ones; this fact supports the chosen core-valence partition and proves the high quality of the core potentials. The use of the valence basis set with a contraction [3/3/4/2] leads to very good results; this contraction scheme reaches a good balance between the quality of the results and the economy of the calculations which are desirable when an ECP method is used and, in consequence, it is advisable. Besides, Table VII shows that if a larger agreement with the AE results is required, it can be accomplished by releasing the outermost primitives of the $p, d$, and $s$ blocks (in this order). We will use a [3/3/4/2] contraction in the rest of the paper.

In order to check the ability of the present core potentials to represent relativistic effects in molecules of third-series transition metal elements, we performed nonrelativistic and relativistic calculations on the ${ }^{2} \Sigma^{+}$ground state of $\mathrm{AuH}^{+}$. The relativistic effects on the bond distance are known to be very large in this molecule and coupled to the electron correlation effects: the bond shortening due to relativistic effects is about $1 \AA$ at noncorrelated levels of calculation ${ }^{44}$ and about $0.4 \AA$ when correlation is also taken into account. ${ }^{45}$
TABLE VI. Atomic spectrum of Pt. All numbers in $\mathrm{cm}^{-1}$.

\begin{tabular}{lccccc}
\hline \hline J & $\begin{array}{c}\text { Main } \\
\text { term }\end{array}$ & DHF+CI & $\begin{array}{c}\text { WB-AIMP } \\
\text { MRCI(SD) }\end{array}$ & $\begin{array}{c}\text { sfss-WB-AIMP } \\
\text { MRCI(S) }\end{array}$ & Experiment $^{\mathrm{c}}$ \\
\hline 3 & ${ }^{3} \mathrm{D}$ & 0 & 0 & 0 & 0 \\
2 & ${ }^{3} \mathrm{D}$ & 1170 & 1040 & 885 & 776 \\
4 & ${ }^{3} \mathrm{~F}$ & 2113 & 1266 & 795 & 824 \\
0 & $\mathrm{a}{ }^{1} \mathrm{~S}$ & 14695 & 9162 & 6079 & 6140 \\
2 & ${ }^{3} \mathrm{P}$ & 7469 & 7735 & 6929 & 6566 \\
3 & ${ }^{3} \mathrm{~F}$ & 11114 & 11292 & 10590 & 10117 \\
1 & ${ }^{3} \mathrm{D}$ & 9727 & 10993 & 10563 & 10132 \\
2 & ${ }^{1} \mathrm{D}$ & - & 15260 & 13517 & 13496 \\
2 & ${ }^{3} \mathrm{~F}$ & - & 17793 & 16068 & 15502 \\
0 & ${ }^{3} \mathrm{P}$ & - & 20382 & 17402 & - \\
1 & ${ }^{3} \mathrm{P}$ & 21704 & 22083 & 19159 & 18567 \\
4 & ${ }^{1} \mathrm{G}$ & 25648 & 26100 & 22440 & 21967 \\
2 & $\mathrm{~b}^{1} \mathrm{D}$ & 28729 & 29883 & 27757 & 26639 \\
0 & $\mathrm{~b}^{1} \mathrm{~S}$ & - & 52636 & 48738 & - \\
\hline \hline
\end{tabular}

${ }^{a}$ Dirac-Hartree-Fock (DHF) plus four-component CI (singles and doubles) calculation from Ref. 41.

${ }^{\mathrm{b}}$ Reference 40.

${ }^{\mathrm{c}}$ Reference 37.

We show in Table VIII that the relativistic effects estimated from the CG-AIMP and NR-AIMP calculations reasonably agree with the results of noncorrelated Dirac-Fock and correlated no-pair spin-free relativistic all-electron calculations. In particular, the fact that the nonrelativistic potential energy curve of $\mathrm{AuH}^{+}\left({ }^{2} \Sigma^{+}\right)$is extremely flat while the relativistic one is not, which is the reason for the large relativistic effects on $R_{e}$, is well reproduced by the AIMP calculations. The coupling between the spin-free relativistic and correlation effects is well reproduced too.

In Table IX we show the results of our CG-AIMP calculations on the MH hydrides ( $\mathrm{M}$ being a transition element of the third series) together with the very scarce experimental information available and other theoretical calculations from the literature in which different valence-only and all-electron spin-free relativistic Hamiltonians were used. In order to summarize, we included only calculations that handled correlation effects at a level which could be compared with our calculations. Most of them are more or less equivalent to our ACPF-ds calculations. In Table IX, MCPF stands for modified coupled pair functional calculations,${ }^{46}$ an approximately size-consistent single-reference CI procedure. SOCI stands for second-order CI, which corresponds to single and double excitations from a CAS multireference and uses, in consequence, the same CI space as our ACPF-ds calculations. $\mathrm{MRCI}(\mathrm{SD})+\mathrm{Q}$ stands for multireference CI with single and double excitations (a CI space equivalent to those of the SOCI and ACPF calculations) with the size-consistency correction of Langhoff and Davidson. ${ }^{47}$ MP4(SDTQ) stands for full fourth order Möller-Plesset perturbation theory calculations. CISD is singles and doubles CI with a size-consistency correction.

Our CASSCF, ACPF-ds and ACPF-[p]ds calculations along the series show a uniform, significant effect of the dynamical correlation of the $d$ and $s$ shells and a minor effect of the $5 p$ correlation, this being more important at the beginning of the series where the participation of the $5 p$ orbital in bonding is expected to be higher. The comparison with 
TABLE VII. Spectroscopic constants of $\mathrm{PtH}^{2} \Sigma^{+}$. Nonrelativistic CASSCF calculations. Distances in $\AA$, vibrational frequencies in $\mathrm{cm}^{-1}$, and dissociation energies in $\mathrm{kcal} / \mathrm{mol}$.

\begin{tabular}{lcccc}
\hline \hline & Pt basis set & $R_{e}$ & $\omega_{e}$ & $D_{e}$ \\
\hline All-electron calculation & & & & \\
& AE-[8/6/6/3] $]^{\mathrm{a}}$ & 1.655 & 1710 & 58.14 \\
NR-AIMP calculations & & & & \\
& AE-[8/6/6/3] $]^{\mathrm{a}}$ & 1.655 & 1720 & 58.15 \\
& & & & \\
& VAL-[3/3/4/2] & 1.662 & 1690 & 56.07 \\
& & & & \\
& VAL-[3/3/4/3] & 1.662 & 1690 & 56.07 \\
& VAL-[3/3/5/2] & 1.659 & 1700 & 56.35 \\
& VAL-[3/4/4/2] & 1.657 & 1710 & 57.39 \\
& VAL-[4/3/4/2] & 1.660 & 1730 & 56.91 \\
& VAL-[4/4/4/2] & 1.656 & 1740 & 58.16 \\
& VAL-[3/4/5/2] & 1.655 & 1720 & 57.70 \\
& VAL-[4/4/5/2] & 1.653 & 1740 & 58.47 \\
\hline \hline
\end{tabular}

${ }^{\mathrm{a} A E}$ stands for all-electron basis set from Ref. 43 .

${ }^{\mathrm{b}} \mathrm{VAL}$ for valence basis set from this work.

experiments is limited to $\mathrm{WH}, \mathrm{AuH}$ and $\mathrm{HgH}$, the only hydrides whose spectroscopic constants have been measured, to our knowledge. The agreement of our results with the measurements of Ref. 48 on $\mathrm{AuH}$ and $\mathrm{HgH}$ is good. Furthermore, in $\mathrm{AuH}$, our ACPF-[p]ds results are very close to the no-pair all-electron coupled-cluster calculations of Ref. 45 in which the same degree of electron correlation had been included. We take these agreements as indications of the good performance of our core potentials. In $\mathrm{HgH}$, the spectroscopic constants have been deduced from measurements in Ref. 49 as well: The bond distance shows some deviation from our results and from the experimental data of Ref. 48; we do not have an explanation for this.

The apparently very large deviations with respect to the experimental bond distance and vibrational frequency of $\mathrm{WH}$ deserve some comments. These data were deduced from the analysis of the emission spectra of a plasma generated by a discharge in Ref. 50. In those experiments the emissions to two different vibrational levels of the electronic ground state were not identified. Instead, the value of $\omega_{e}$ was obtained as a rough estimate from the effective rotational constant $\mathrm{B}_{v}$ and the effective centrifugal constant $\mathrm{D}_{v}$, with $v$ unknown. This means that very large deviations from the true vibrational constant are possible: The agreement between our calculations and those of Ref. 51 indicate that this should be the case. Also, the too low values of $\mathrm{R}_{e}$ obtained in all the available theoretical calculations, in contrast with what is usual and with the results on $\mathrm{AuH}$ and $\mathrm{HgH}$, might indicate that the interpretation of the emission spectrum measurements in Ref. 50 is questionable. In particular, a large value of $v$ for the effective rotational constant $\mathrm{B}_{v}$ has not been ruled out; if this were so, the $\mathrm{R}_{e}$ obtained from $\mathrm{B}_{v}$ in Ref. 50 would be significantly overestimated.

Overall, our results are similar to those of other methods. In particular, the closeness between our CG-AIMP ACPF-ds results and the NP-AIMP MCPF results of Wittborn and Wahlgren, ${ }^{19}$ which correspond to the spin-free no-pair Hamiltonian of Douglas-Kroll ${ }^{18}$ is remarkable. Since both CG-AIMP and NP-AIMP methods have a common imple-
TABLE VIII. Spectroscopic constants of the ${ }^{2} \Sigma^{+}$ground state of $\mathrm{AuH}^{+}$. Distances in $\AA$, vibrational frequencies in $\mathrm{cm}^{-1}$, and dissociation energies in $\mathrm{kcal} / \mathrm{mol}$. Dissociation limit is $\mathrm{Au}^{+}\left(5 d^{10}-{ }^{1} \mathrm{~S}\right)+\mathrm{H}\left({ }^{2} \mathrm{~S}\right)$. Numbers in parentheses correspond to the relativistic effects.

\begin{tabular}{|c|c|c|c|c|}
\hline Method & Reference & $R_{e}$ & $\omega_{e}$ & $D_{e}$ \\
\hline All-electron nonrel. HF & 44 & 2.57 & & \\
\hline All-electron $\mathrm{DHF}^{\mathrm{a}}$ & 44 & $1.56(-1.01)$ & 2200 & 13.6 \\
\hline All-electron nonrel. CC & 45 & 1.936 & 600 & - \\
\hline All-electron no-pair rel. $\mathrm{CC}^{\mathrm{b}}$ & 45 & $1.531(-0.41)$ & 2238 & $45^{\mathrm{c}}$ \\
\hline EAPP $^{\mathrm{d}}$ nonrel. HF & 59 & 2.636 & 305 & 1.6 \\
\hline $\mathrm{EAPP}^{\mathrm{d}}$ rel. HF & 59 & $1.549(-1.09)$ & 2124 & 10.4 \\
\hline $\mathrm{EAPP}^{\mathrm{d}}$ rel. CISD+Q & 59 & 1.501 & 2312 & 39.8 \\
\hline NR-AIMP CASSCF & $\cdots$ & 2.469 & 690 & 1.4 \\
\hline CG-AIMP CASSCF & $\cdots$ & $1.571(-0.90)$ & 1960 & 24.1 \\
\hline NR-AIMP ACPF-ds & $\cdots$ & 2.300 & 401 & 3.5 \\
\hline CG-AIMP ACPF-ds & $\cdots$ & $1.541(-0.76)$ & 2040 & 34.5 \\
\hline NR-AIMP ACPF-[p]ds & $\cdots$ & 2.185 & 434 & 4.2 \\
\hline CG-AIMP ACPF-[p]ds & $\cdots$ & $1.531(-0.65)$ & 2082 & 39.6 \\
\hline
\end{tabular}

${ }^{\mathrm{a}} \Gamma_{1 / 2}$ state.

${ }^{\mathrm{b}}$ Coupled-cluster (CC) calculations starting from $\mathrm{AuH}$ reference.

${ }^{\mathrm{c}}$ Coupled-cluster calculations starting from $\mathrm{AuH}^{++}$reference.

${ }^{\mathrm{d}}$ Energy-adjusted pseudopotentials from Ref. 9 corresponding to the core $[\mathrm{Kr}, 4 \mathrm{~d}, 4 \mathrm{f}]$

mentation of the frozen-core approximations involved, the previous agreement indicates that the Douglas-Kroll and Cowan-Griffin Hamiltonians are of similar quality for these molecular calculations, in spite of their different origins. The existence of systematic deviations with other methods is not apparent, except for a tendency to produce $\omega_{e}$ values slightly smaller than those of spin-free averaged relativistic ECP $(\mathrm{AREP})^{8}$ calculations. With respect to the disagreement in the ground state of the $\mathrm{ReH}$ molecule found by Wittborn and Wahlgren ${ }^{19}\left({ }^{5} \Sigma^{+}\right)$and Dai and Balasubramanian ${ }^{52}\left({ }^{7} \Sigma^{+}\right)$, our calculations agree with the former result. We find another disagreement in the ground state of $\mathrm{IrH}$ according to our calculation $\left({ }^{3} \Delta\right)$ and to those of Dai and Balasubramanian ${ }^{53}\left({ }^{3} \Sigma^{-}\right)$at an equivalent level of correlation; the energy difference between the two states is, however, small.

\section{CONCLUSIONS}

We produced and monitored the quality of the ingredients of relativistic spin-free CG-AIMP and spin-orbit WBAIMP calculations with La and third-series transition metal elements. Starting from atomic Cowan-Griffin calculations, we generated the spin-free relativistic core AIMPs which correspond to the 62 -electron core $[\mathrm{Cd}, 4 f]$. Of those elements, we obtained their spin-orbit operators and their corresponding optimized, spin-orbit-corrected valence basis sets. We produced, as well, the nonrelativistic AIMPs and valence basis sets, since they are necessary for the explicit calculation of the relativistic effects. Also, we performed monitoring spin-free calculations on the atoms, singly ionized ions, and monohydrides of the ten elements, which revealed the good quality of the AIMPs. Finally, we carried out a spin-free-state-shifted spin-orbit-CI calculation on the 
TABLE IX. Spectroscopic constants of third-series transition metal hydrides. Spin-free relativistic calculations and experimental results. Distances in $\AA$, vibrational frequencies in $\mathrm{cm}^{-1}$, and dissociation energies in $\mathrm{kcal} /$ mol.

\begin{tabular}{|c|c|c|c|c|c|c|c|c|}
\hline Molecule & State & Method & Level & Reference & $R_{e}$ & $\omega_{e}$ & $D_{e}$ & Dissoc. limit \\
\hline \multirow[t]{6}{*}{$\mathrm{LaH}^{\mathrm{a}}$} & \multirow[t]{6}{*}{${ }^{1} \Sigma^{+}$} & \multirow[t]{3}{*}{ CG-AIMP $^{b}$} & CASSCF & $\cdots$ & 2.11 & 1350 & 61.7 & \multirow[t]{6}{*}{$\mathrm{La}\left(s^{2} d^{1}\right)^{2} D$} \\
\hline & & & ACPF-ds & $\cdots$ & 2.08 & 1380 & 63.2 & \\
\hline & & & ACPF-[p]ds & $\cdots$ & 2.06 & 1420 & 62.6 & \\
\hline & & NP-AIMP $^{c}$ & MCPF & 19 & 2.08 & - & 63.7 & \\
\hline & & $N P-A E^{d}$ & $\mathrm{MCPF}$ & 19 & 2.07 & - & 64.2 & \\
\hline & & $\mathrm{AREP}^{\mathrm{e}}$ & SOCI & 33 & 2.08 & 1433 & 60.0 & \\
\hline \multirow[t]{6}{*}{$\mathrm{HfH}$} & \multirow[t]{6}{*}{${ }^{2} \Delta$} & \multirow[t]{3}{*}{ CG-AIMP $^{b}$} & CASSCF & $\cdots$ & 1.84 & 1730 & 54.2 & \multirow[t]{6}{*}{$\operatorname{Hf}\left(s^{2} d^{2}\right)^{3} F$} \\
\hline & & & ACPF-ds & $\cdots$ & 1.84 & 1680 & 64.2 & \\
\hline & & & ACPF-[p]ds ${ }^{j}$ & & 1.82 & 1710 & 62.6 & \\
\hline & & NP-AIMP & MCPF & 19 & 1.85 & - & 64.4 & \\
\hline & & $N P-A E^{d}$ & MCPF & 19 & 1.86 & - & 65.2 & \\
\hline & & $\mathrm{AREP}^{\mathrm{e}}$ & SOCI & 60 & 1.85 & 1702 & 66.2 & \\
\hline \multirow[t]{5}{*}{$\mathrm{TaH}$} & \multirow[t]{5}{*}{${ }^{3} \Phi$} & CG-AIMP $^{b}$ & CASSCF & & 1.77 & 1730 & 43.8 & \multirow[t]{5}{*}{$\mathrm{Ta}\left(s^{2} d^{3}\right){ }^{4} F$} \\
\hline & & & ACPF-ds & & 1.75 & 1800 & 56.7 & \\
\hline & & NP-AIMP $^{c}$ & MCPF & 19 & 1.76 & - & 55.2 & \\
\hline & & $N P-A E^{d}$ & MCPF & 19 & 1.76 & - & 57.8 & \\
\hline & & $\mathrm{AREP}^{\mathrm{e}}$ & SOCI & 61 & 1.75 & 1810 & 55.1 & \\
\hline \multirow[t]{7}{*}{ WH } & \multirow[t]{7}{*}{${ }^{6} \Sigma^{+}$} & \multirow[t]{3}{*}{ CG-AIMP $^{\mathrm{b}}$} & CASSCF & & 1.76 & 1730 & 46.5 & \multirow[t]{7}{*}{$\mathrm{W}\left(s^{1} d^{5}\right)^{7} S$} \\
\hline & & & ACPF-ds & & 1.72 & 1820 & 61.3 & \\
\hline & & & ACPF-[p]ds & & 1.71 & 1820 & 62.9 & \\
\hline & & NP-AIMP $^{c}$ & $\mathrm{MCPF}$ & 19 & 1.73 & - & 61.9 & \\
\hline & & $N P-A E^{d}$ & MCPF & 19 & 1.73 & - & 62.9 & \\
\hline & & $\mathrm{AREP}^{\mathrm{e}}$ & SOCI & 51 & 1.73 & 1897 & 62.0 & \\
\hline & & Experiment $\mathrm{f}^{\mathrm{f}}$ & & 50 & 1.79 & 531 & - & \\
\hline \multirow[t]{11}{*}{$\mathrm{ReH}$} & ${ }^{5} \Sigma^{+}$ & CG-AIMP $^{b}$ & CASSCF & & 1.67 & 1920 & 24.1 & $\operatorname{Re}\left(s^{2} d^{5}\right){ }^{6} S$ \\
\hline & & & ACPF-ds & & 1.64 & 1970 & 43.7 & \\
\hline & & & ACPF-[p]ds & & 1.64 & 1950 & 46.2 & \\
\hline & & NP-AIMP ${ }^{c}$ & MCPF & 19 & 1.64 & - & 45.3 & \\
\hline & & $N P-A E^{d}$ & $\mathrm{MCPF}$ & 19 & 1.64 & - & 44.8 & \\
\hline & & $\mathrm{AREP}^{\mathrm{e}}$ & SOCI & 52 & 1.63 & 2042 & 22.1 & \\
\hline & ${ }^{7} \Sigma^{+}$ & CG-AIMP ${ }^{b}$ & CASSCF & & 1.82 & 1550 & 29.8 & $\operatorname{Re}\left(s^{2} d^{5}\right){ }^{6} S$ \\
\hline & & & ACPF-ds & & 1.80 & 1520 & 36.6 & \\
\hline & & & ACPF-[p]ds & & 1.79 & 1550 & 36.1 & \\
\hline & & NP-AIMP ${ }^{c}$ & MCPF & 19 & 1.81 & - & 24.8 & \\
\hline & & $\mathrm{AREP}^{\mathrm{e}}$ & SOCI & 52 & 1.82 & 1611 & 30.4 & \\
\hline OsH & ${ }^{4} \Pi$ & CG-AIMP ${ }^{b}$ & CASSCF & & 1.63 & 1960 & 39.8 & Os $\left(s^{2} d^{6}\right){ }^{5} D$ \\
\hline & & & ACPF-ds & & 1.58 & 2130 & 58.2 & \\
\hline & & & ACPF-[p]ds & & 1.58 & 2160 & 59.5 & \\
\hline & & NP-AIMP ${ }^{c}$ & MCPF & 19 & 1.59 & - & 61.5 & \\
\hline & & $N P-A E^{d}$ & $\mathrm{MCPF}$ & 19 & 1.59 & - & 60.5 & \\
\hline & & $\mathrm{AREP}^{\mathrm{e}}$ & SOCI & 62 & 1.58 & 2212 & - & \\
\hline & & $\mathrm{EAPP}^{\mathrm{g}}$ & $\mathrm{MRCI}(\mathrm{SD})+\mathrm{Q}$ & 63 & 1.56 & 2327 & 62.7 & \\
\hline & & $\mathrm{HW}-\mathrm{ECP}^{\mathrm{h}}$ & $\operatorname{MRCI}(\mathrm{SD})+\mathrm{Q}$ & 63 & 1.56 & 2339 & 63.0 & \\
\hline $\mathrm{IrH}$ & ${ }^{3} \Delta$ & CG-AIMP ${ }^{b}$ & CASSCF & & 1.60 & 2010 & 52.4 & $\operatorname{Ir}\left(s^{2} d^{7}\right)^{4} F$ \\
\hline & & & ACPF-ds & & 1.55 & 2230 & 73.7 & \\
\hline & & & ACPF-[p]ds & & 1.54 & 2270 & 70.8 & \\
\hline & & NP-AIMP ${ }^{c}$ & $\mathrm{MCPF}$ & 19 & 1.57 & - & 76.9 & \\
\hline & & $N P-A E^{d}$ & $\mathrm{MCPF}$ & 19 & 1.55 & - & 74.4 & \\
\hline & & $\mathrm{AREP}^{\mathrm{e}}$ & $\operatorname{MRCI}(\mathrm{SD})$ & 53 & 1.55 & 2316 & 58.3 & \\
\hline & ${ }^{3} \Sigma^{-}$ & CG-AIMP ${ }^{b}$ & CASSCF & & 1.59 & 2030 & 48.3 & $\operatorname{Ir}\left(s^{2} d^{7}\right)^{4} F$ \\
\hline & & & ACPF-ds & & 1.54 & 2230 & 69.6 & \\
\hline & & & ACPF-[p]ds & & 1.53 & 2210 & 69.4 & \\
\hline & & $\mathrm{AREP}^{\mathrm{e}}$ & $\operatorname{MRCI}(\mathrm{SD})$ & 53 & 1.56 & 2476 & 60.9 & \\
\hline PtH & ${ }^{2} \Sigma^{+}$ & CG-AIMP & CASSCF & & 1.58 & 2050 & 55.2 & $\operatorname{Pt}\left(s^{1} d^{9}\right){ }^{3} D$ \\
\hline & & & ACPF-ds & & 1.52 & 2290 & 77.5 & \\
\hline & & & ACPF-[p]ds & & 1.52 & 2310 & 78.0 & \\
\hline & & NP-AIMP ${ }^{c}$ & $\mathrm{MCPF}$ & 19 & 1.52 & - & 74.0 & \\
\hline
\end{tabular}


TABLE IX. (Continued.)

\begin{tabular}{|c|c|c|c|c|c|c|c|c|}
\hline Molecule & State & Method & Level & Reference & $R_{e}$ & $\omega_{e}$ & $D_{e}$ & Dissoc. limit \\
\hline & & $N P-A E^{d}$ & MCPF & 19 & 1.51 & - & 74.0 & \\
\hline & & HW-ECP ${ }^{h}$ & MP4(SDTQ) & 64 & 1.50 & 2544 & 75.4 & \\
\hline & & $\mathrm{RECP}^{\mathrm{i}}$ & $\operatorname{MRCI}(\mathrm{SD})$ & 65 & 1.54 & - & 71.7 & \\
\hline & $\Omega=\frac{1}{2}$ & 4-comp. AE & $\mathrm{DHF}+\mathrm{CI}^{\mathrm{k}}$ & 41 & 1.53 & 2419 & 63.2 & \\
\hline \multirow[t]{7}{*}{$\mathrm{AuH}$} & ${ }^{1} \Sigma^{+}$ & CG-AIMP ${ }^{b}$ & CASSCF & $\cdots$ & 1.59 & 1910 & 49.1 & $\mathrm{Au}\left(s^{1} d^{10}\right)^{2} S$ \\
\hline & & & ACPF-ds & $\cdots$ & 1.55 & 2120 & 65.0 & \\
\hline & & & ACPF-[p]ds & $\cdots$ & 1.53 & 2140 & 68.0 & \\
\hline & & $N P-A E^{d}$ & $\mathrm{CC}[\mathrm{p}] \mathrm{ds}$ & 45 & 1.52 & 2288 & - & \\
\hline & & $\mathrm{AREP}^{\mathrm{e}}$ & CISD & 66 & 1.59 & 2100 & - & \\
\hline & & EAPP $^{g}$ & CISD & 59 & 1.50 & 2288 & 68.2 & \\
\hline & & Experiment & & 48 & 1.52 & 2303 & 74.3 & \\
\hline \multirow[t]{8}{*}{$\mathrm{HgH}$} & ${ }^{2} \Sigma^{+}$ & CG-AIMP ${ }^{b}$ & CASSCF & $\cdots$ & 1.81 & 1200 & -8.8 & $\mathrm{Hg}\left(s^{2} d^{10}\right){ }^{1} S$ \\
\hline & & & ACPF-ds & $\cdots$ & 1.80 & 1110 & 9.8 & \\
\hline & & & ACPF-[p]ds & $\cdots$ & 1.78 & 1270 & 10.4 & \\
\hline & & $\mathrm{AREP}^{\mathrm{e}}$ & MRD-CI & 67 & 1.78 & 1309 & 7.3 & \\
\hline & & EAPP & CISD & 68 & 1.73 & 1185 & 2.8 & \\
\hline & & & QCI & $\ldots$ & 1.79 & 1156 & 6.9 & \\
\hline & & Experiment & & 48 & 1.77 & 1203 & 10.4 & \\
\hline & & & & 49 & 1.74 & 1387 & 10.6 & \\
\hline
\end{tabular}

a [Cd]-core and [3/3/3/1] valence basis set for La.

${ }^{\mathrm{b}} \mathrm{Spin}$-free relativistic CG-AIMP calculations of this work corresponding to the core $[\mathrm{Cd}, 4 \mathrm{f}]$.

${ }^{\mathrm{c} N o-p a i r}$ spin-free relativistic AIMP from Ref. 19 corresponding to the core [Ar,3d].

${ }^{\mathrm{d}}$ No-pair spin-free relativistic all-electron calculations.

${ }^{\mathrm{e}} \mathrm{Spin}-$ free averaged relativistic ECP from Ref. 8 corresponding to the core $[\mathrm{Kr}, 4 \mathrm{~d}, 4 \mathrm{f}]$.

${ }^{\mathrm{f}} \mathrm{See}$ text for a discussion.

${ }^{\mathrm{g}}$ Spin-free relativistic energy-adjusted pseudopotential from Ref. 9 corresponding to the core $[\mathrm{Kr}, 4 \mathrm{~d}, 4 \mathrm{f}]$.

${ }^{\mathrm{h}}$ Spin-free relativistic ECP from Ref. 7 corresponding to the core $[\mathrm{Kr}, 4 \mathrm{~d}, 4 \mathrm{f}]$.

${ }^{i}$ Spin-free relativistic ECP from Ref. 3.

${ }^{\mathrm{j}}$ Here the reference space is a selection of the CAS: only configurations with coefficients with an absolute value larger than 0.05 are retained.

${ }^{\mathrm{k}}$ Dirac-Hartree-Fock plus four-component CI calculations.

even spectrum of Pt which confirmed the conclusions of Ref. 26: The quality of the one-electron WB-AIMP spin-orbit operators is very high; thus, the quality of the spin-orbit splittings in WB-AIMP calculations is mainly limited by the treatment of the correlation effects, which can be handled at the spin-free level with standard $a b$ initio techniques with a nonrelativistic structure.

\section{ACKNOWLEDGMENT}

This work was partly supported by a grant from the Ministerio de Educación y Cultura of Spain (Grant No. PB950201).

\section{APPENDIX}

The numerical all-electron atomic nonrelativistic Hartree-Fock and spin-free relativistic Cowan-GriffinHartree-Fock calculations necessary to produce the core orbitals have been performed with the program MCHF72. ${ }^{54}$ The optimization of the valence basis sets has been carried out with a modified version of the program $\mathrm{ABS}^{55}$ adapted to handle AIMP integrals. The spin-free CASSCF and ACPF calculations have been performed with the MOLCAS.4 package. ${ }^{28}$ We used a modified version of the COLUMBus suite of programs ${ }^{56}$ for the spin-orbit-CI calculations; the AIMP integrals are calculated with ECPAIMP ${ }^{57}$ in this case.
${ }^{1}$ M. Krauss and W. J. Stevens, Annu. Rev. Phys. Chem. 35, 357 (1984).

${ }^{2}$ S. Huzinaga, Can. J. Chem. 73, 619 (1995).

${ }^{3}$ O. Gropen, in Relativistic Effects in Atoms and Molecules, Vol. 2 of Methods in Computational Chemistry, edited by S. Wilson (Plenum, New York, 1988), p. 109.

${ }^{4}$ J. C. Phillips and L. Kleinman, Phys. Rev. 116, 287 (1959).

${ }^{5}$ G. B. Bachelet, D. R. Hamann, and M. Schlüter, Phys. Rev. B 26, 4199 (1982).

${ }^{6}$ P. J. Hay and W. R. Wadt, J. Chem. Phys. 82, 270 (1985).

${ }^{7}$ P. J. Hay and W. R. Wadt, J. Chem. Phys. 82, 299 (1985).

${ }^{8}$ R. B. Ross, J. M. Powers, T. Atashroo, W. C. Ermler, L. A. LaJohn, and P. A. Christiansen, J. Chem. Phys. 93, 6654 (1990).

${ }^{9}$ D. Andrae, U. Häußermann, M. Dolg, H. Stoll, and H. Preuss, Theor. Chim. Acta 77, 123 (1990).

${ }^{10}$ S. Huzinaga and A. A. Cantu, J. Chem. Phys. 55, 5543 (1971).

${ }^{11}$ Y. Sakai, E. Miyoshi, M. Klobukowski, and S. Huzinaga, J. Comput. Chem. 8, 226 (1987); 8, 256 (1987).

${ }^{12}$ S. Huzinaga, L. Seijo, Z. Barandiarán, and M. Klobukowski, J. Chem. Phys. 86, 2132 (1987).

${ }^{13}$ R. D. Cowan and D. C. Griffin, J. Opt. Soc. Am. 66, 1010 (1976).

${ }^{14}$ Z. Barandiarán and L. Seijo, Can. J. Chem. 70, 409 (1992).

${ }^{15}$ Z. Barandiarán and L. Seijo, J. Chem. Phys. 101, 4049 (1994).

${ }^{16}$ L. Seijo, Z. Barandiarán, and S. Huzinaga, J. Chem. Phys. 91, 7011 (1989).

${ }^{17}$ Z. Barandiarán, L. Seijo, and S. Huzinaga, J. Chem. Phys. 93, 5843 (1990).

${ }^{18}$ M. Douglas and N. M. Kroll, Ann. Phys. (N.Y.) 82, 89 (1974).

${ }^{19}$ C. Wittborn and U. Wahlgren, Chem. Phys. 201, 357 (1995).

${ }^{20}$ F. Rakowitz, C. M. Marian, L. Seijo, and U. Wahlgren, J. Chem. Phys. (in press).

${ }^{21}$ J. H. Wood and A. M. Boring, Phys. Rev. B 18, 2701 (1978).

${ }^{22}$ S. Katsuki and S. Huzinaga, Chem. Phys. Lett. 147, 597 (1988).

${ }^{23}$ L. Seijo, J. Chem. Phys. 102, 8078 (1995). 
${ }^{24}$ M. Casarrubios and L. Seijo, J. Mol. Struct.: THEOCHEM 426, 59 (1998).

${ }^{25}$ R. Llusar, M. Casarrubios, Z. Barandiarán, and L. Seijo, J. Chem. Phys. 105, 1 (1996)

${ }^{26}$ F. Rakowitz, M. Casarrubios, L. Seijo, and C. M. Marian, J. Chem. Phys. 108, 7980 (1998).

${ }^{27}$ E. Francisco, L. Seijo, and L. Pueyo, J. Solid State Chem. 63, 391 (1986).

${ }^{28} \mathrm{~K}$. Andersson et al. MOLCAS version 4, Lund University, Sweden, 1997.

${ }^{29}$ Detailed core AIMP and valence basis set data libraries in electronic format are available from the authors upon request or directly at the address http://www.qui.uam.es/Data/AIMPLibs.html. See also Ref. 28.

${ }^{30}$ See AIP Document No. PAPS JCPSA6-110-303901 for 32 pages of Valence Gaussian basis sets, Coulomb model potentials, and core orbitals. Order by PAPS number and journal reference from American Institute of Physics, Physics Auxiliary Publication Service, 500 Sunnyside Boulevard, Woodbury, NY 11797-2999. Fax: 516-576-2223, e-mail: paps@aip.org. The price is $\$ 1.50$ for each microfiche (98 pages) or $\$ 5.00$ for photocopies of up to 30 pages, and $\$ 0.15$ for each additional page over 30 pages. Airmail additional. Make checks payable to the American Institute of Physics.

${ }^{31}$ R. M. Pitzer and N. W. Winter, J. Phys. Chem. 92, 3061 (1988).

${ }^{32}$ Gaussian Basis Sets for Molecular Calculations, edited by S. Huzinaga (Elsevier, Amsterdam, 1984).

${ }^{33}$ K. K. Das and K. Balasubramanian, Chem. Phys. Lett. 172, 373 (1990).

${ }^{34}$ P. J. Hay, J. Chem. Phys. 66, 4377 (1977).

${ }^{35}$ B. O. Roos, P. R. Taylor, and P. E. M. Siegbahn, Chem. Phys. 48, 157 (1980); P. E. M. Siegbahn, A. Heiberg, J. Almlöf, and B. O. Roos, J. Chem. Phys. 74, 2384 (1981); P. E. M. Siegbahn, A. Heiberg, B. Roos, and B. Levy, Phys. Scr. 21, 323 (1980).

${ }^{36}$ R. Ahlrichs, P. Scharf, and C. Ehrhardt, J. Chem. Phys. 82, 890 (1985); R. J. Gdanitz and R. Ahlrichs, Chem. Phys. Lett. 143, 413 (1988).

${ }^{37}$ C. E. Moore, Atomic Energy Levels (National Bureau of Standards, Washington, D. C., 1952), circular 467, Vols 2 and 3 (1959).

${ }^{38}$ R. D. Cowan, The Theory of Atomic Structure and Spectra (University of California Press, Berkeley, 1981).

${ }^{39}$ T. van Kleef and B. Metsch, Physica B \& C 95, 251 (1978).

${ }^{40}$ M. Casarrubios and L. Seijo, Chem. Phys. Lett. 236, 510 (1995).

${ }^{41}$ L. Visscher, T. Saue, W. C. Nieuwpoort, K. Faegri, and O. Gropen, J. Chem. Phys. 99, 6704 (1998).

${ }^{42}$ S. Huzinaga, J. Chem. Phys. 42, 1293 (1965).

${ }^{43} \mathrm{~K}$. Faegri (private communication).
${ }^{44}$ Y. Ishikawa, G. L. Malli, and N. C. Pyper, 194, 481 (1992).

${ }^{45}$ U. Kaldor and B. A. Hess, Chem. Phys. Lett. 230, 1 (1994).

${ }^{46}$ D. P. Chong and S. R. Langhoff, J. Chem. Phys. 84, 5606 (1986).

${ }^{47}$ S. R. Langhoff and E. R. Davidson, Int. J. Quantum Chem. 8, 61 (1974).

${ }^{48}$ K. P. Huber and G. Herzberg, Molecular Spectra and Molecular Structure of Diatomic Molecules (Van Nostrand, New York, 1979).

${ }^{49}$ W. C. Stwalley, J. Chem. Phys. 63, 3062 (1975).

${ }^{50}$ J. F. Garvey and A. Kuppermann, J. Phys. Chem. 92, 4583 (1988).

${ }^{51}$ Z. Ma and K. Balasubramanian, Chem. Phys. Lett. 181, 467 (1991).

${ }^{52}$ D. Dai and K. Balasubramanian, J. Mol. Spectrosc. 158, 455 (1993).

${ }^{53}$ D. Dai and K. Balasubramanian, New J. Chem. 15, 721 (1991).

${ }^{54}$ C. Froese-Fischer, The Hartree-Fock Method for Atoms (Wiley, New York, 1977); MCHF72 program, Version modified by M. Klobukowski and L. Seijo.

${ }^{55}$ S. Huzinaga, M. Klobukowski, and Y. Sakai, Comput. Phys. Commun. 30, 311 (1983).

${ }^{56}$ R. M. Pitzer (principal author), COLUMBUs suite of programs (ARGOS, CNVRT, SCFPQ, LSTRN, CGDBG, and CIDBG); See: A. H. H. Chang and R. M. Pitzer, J. Am. Chem. Soc. 111, 2500 (1989), and references therein for a description. CNVRT and LSTRN have been adapted to handle ECPAIMP integrals by L. Seijo. CIDBG has been modified for spin-free-state-shifted spin-orbit CI calculations by M. Casarrubios.

${ }^{57}$ ECPAIMP is an integral program for ECP and AIMP calculations written by L. G. M. Pettersson, L. Seijo, and M. A. Nygren.

${ }^{58}$ R. L. Martin and P. J. Hay, J. Chem. Phys. 75, 4539 (1981).

${ }^{59}$ P. Schwerdtfeger, M. Dolg, W. H. E. Schwarz, G. A. Bowmaker, and P. D. W. Boyd, J. Chem. Phys. 91, 1762 (1989).

${ }^{60}$ K. Balasubramanian and K. K. Das, J. Mol. Spectrosc. 145, 142 (1991).

${ }^{61}$ W. Cheng and K. Balasubramanian, J. Mol. Spectrosc. 149, 99 (1991).

${ }^{62}$ M. Benavides-Garcia and K. Balasubramanian, J. Mol. Spectrosc. 150, 271 (1991).

${ }^{63}$ D. Andrae, U. Häudßermann, M. Dolg, H. Stoll, and H. Preuss, Theor. Chim. Acta 78, 247 (1991).

${ }^{64}$ C. M. Rohlfing, P. J. Hay, and R. L. Martin, J. Chem. Phys. 85, 1447 (1986).

${ }^{65}$ O. Gropen, J. Almlöf, and U. Wahlgren, J. Chem. Phys. 96, 8363 (1992).

${ }^{66}$ R. B. Ross and W. C. Ermler, J. Phys. Chem. 89, 5202 (1985).

${ }^{67}$ A. B. Alekseyev, H.-P. Liebermann, R. J. Buenker, and G. Hirsch, J. Chem. Phys. 104, 4672 (1996).

${ }^{68}$ U. Haussermann, M. Dolg, H. Stoll, H. Preuss, P. Schwerdtfeger, and R. M. Pitzer, Mol. Phys. 78, 1211 (1993). 